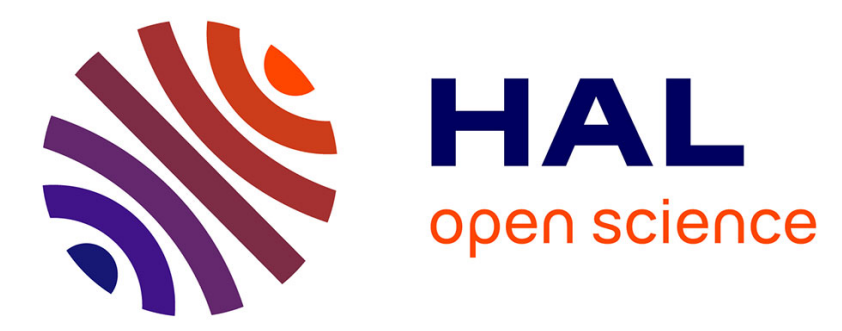

\title{
Comparison of Different Definitions of Traces for a Class of Ramified Domains with Self-Similar Fractal Boundaries
}

\author{
Yves Achdou, Thibaut Deheuvels, Nicoletta Tchou
}

\section{- To cite this version: \\ Yves Achdou, Thibaut Deheuvels, Nicoletta Tchou. Comparison of Different Definitions of Traces for a Class of Ramified Domains with Self-Similar Fractal Boundaries. Potential Analysis, 2014, 40 (4), pp.345-362. 10.1007/s11118-013-9352-y · hal-00657954}

\section{HAL Id: hal-00657954 https://hal.science/hal-00657954}

Submitted on 9 Jan 2012

HAL is a multi-disciplinary open access archive for the deposit and dissemination of scientific research documents, whether they are published or not. The documents may come from teaching and research institutions in France or abroad, or from public or private research centers.
L'archive ouverte pluridisciplinaire HAL, est destinée au dépôt et à la diffusion de documents scientifiques de niveau recherche, publiés ou non, émanant des établissements d'enseignement et de recherche français ou étrangers, des laboratoires publics ou privés. 


\title{
Comparison of Different Definitions of Traces for a Class of Ramified Domains with Self-Similar Fractal Boundaries
}

\author{
Yves Achdou * Thibaut Deheuvels $†$ Nicoletta Tchou $\ddagger$
}

November 23, 2011

\begin{abstract}
We consider a class of ramified bidimensional domains $\Omega$ with a self-similar fractal boundary $\Gamma^{\infty}$, which is supplied with a probability measure $\mu$ called the self-similar measure. Emphasis is put on the case when the domain is not a $\epsilon-\delta$ domain as defined by Jones and the fractal set is not totally disconnected. We compare two notions of trace on $\Gamma^{\infty}$ for functions in $W^{1, q}(\Omega)$ : the classical one, see for instance the book by Jonnson and Wallin, 1984, page 206 , using the strict definition of a function at a point of $\bar{\Omega}$, and another one proposed in 2007 and heavily relying on self-similarity. We prove that the two traces coincide $\mu$-almost everywhere on $\Gamma^{\infty}$. As a corollary, we characterize the critical number $\bar{q}$ for which for all $q<\bar{q}$ (resp. $q>\bar{q}$ ) there is a (resp. no) continuous extension operator from $W^{1, q}(\Omega)$ to $W^{1, q}\left(\mathbb{R}^{2}\right)$.
\end{abstract}

\section{Introduction}

In the present work, we consider a class of self-similar sets noted $\Gamma^{\infty}$ below, see for example Figure 1. The set $\Gamma^{\infty}$ is the unique compact subset of $\mathbb{R}^{2}$ such that

$$
\Gamma^{\infty}=f_{1}\left(\Gamma^{\infty}\right) \cup f_{2}\left(\Gamma^{\infty}\right),
$$

where $f_{1}$ and $f_{2}$ are two similitudes with rotation angles $\pm \theta$ and contraction factor $a, 0<a \leq$ $a^{*}(\theta)$. As we shall see, $\Gamma^{\infty}$ can be seen as a part of the boundary of a ramified domain $\Omega$ in $\mathbb{R}^{2}$, see Figure 1, and the restriction $a \leq a^{*}(\theta)$ allows for the construction of $\Omega$ as a union of non-overlapping sub-domains, see (12). In $\S 2.5$, we will recall the notion of self-similar measure $\mu$ defined in the triplet $\left(\Gamma^{\infty}, f_{1}, f_{2}\right)$, see [16]. With the Borel regular probability measure $\mu, \Gamma^{\infty}$ is a $d$-set where $d \equiv-\log 2 / \log a$ is the Hausdorff dimension of $\Gamma^{\infty}$, i.e. there exist two positive constants $c_{1}$ and $c_{2}$ with

$$
c_{1} r^{d} \leq \mu\left(B(x, r) \cap \Gamma^{\infty}\right) \leq c_{2} r^{d}
$$

\footnotetext{
${ }^{*}$ Univ. Paris Diderot, Laboratoire Jacques-Louis Lions, UMR 7598, UPMC, CNRS, F-75205 Paris, France. achdou@math.jussieu.fr

${ }^{\dagger}$ IRMAR, Université de Rennes 1, Rennes, France, thibaut.deheuvels@univ-rennes1.fr

${ }^{\ddagger}$ IRMAR, Université de Rennes 1, Rennes, France, nicoletta.tchou@univ-rennes1.fr
} 
for all $x \in \Gamma^{\infty}$ and $r<1$ (here $B(x, r)$ is the ball with center $x$ and radius $r$ );

Such a geometry can be seen as a bidimensional idealization of the bronchial tree, for example. Indeed, the present work is a continuation of [2] and of [3], which were part of a wider project aimed at simulating the diffusion of medical sprays in lungs. Since the exchanges between the lungs and the circulatory system take place only in the last generations of the bronchial tree (the smallest structures), reasonable models for the diffusion of, e.g., oxygen may involve a non-homogeneous Neumann or Robin condition on the boundary $\Gamma^{\infty}$. Similarly, the lungs are mechanically coupled to the diaphragm, which also implies non-homogeneous boundary conditions on $\Gamma^{\infty}$, if one is interested in a coupled fluid-structure model. It is therefore necessary to study traces of functions on $\Gamma^{\infty}$.

There are several possible ways of defining the trace of a function $v \in W^{1, q}(\Omega)$ on $\Gamma^{\infty}$. The first one, refered to as the classical or strictly defined trace below, relies on the notion of the strict definition of a function at a point $x \in \bar{\Omega}$, see for instance [14] page 206. It is recalled in $\S 4$ below.

For $1 / 2 \leq a \leq a^{*}$, a different notion of trace was intoduced in [2]. Its construction is recalled in $\S 3.3$ below. This trace operator noted $\ell^{\infty}$ below, is obtained by exploiting the self-similarity, as the limit of a sequence of operators $\left(\ell^{n}\right)_{n}$ : the operator $\ell^{n}$ maps $W^{1, q}(\Omega)$ to piecewise constant functions on a partition of $\Gamma^{\infty}$ into $2^{n}$ sets whose $\mu$-measure is $2^{-n}$. Obtained by passing to the limit, $\ell^{\infty}$ is linear and continuous from $W^{1, q}(\Omega)$ to $L^{q}\left(\Gamma^{\infty}, d \mu\right)$. The self-similar construction of the trace operator $\ell^{\infty}$ has permitted to obtain several results:

- The space $\ell^{\infty}\left(W^{1, q}(\Omega)\right)$ was characterized in [3] as a JLip space, see $\S 3.3$ below for the precise statement. The JLip spaces were presented in [12]: Haar wavelets of arbitrary order on $\Gamma^{\infty}$ were used for constructing a family of Lipschitz function spaces allowing jumps at the multiple points in $\Gamma^{\infty}$. These function spaces were named $J \operatorname{Lip}\left(t, p, q ; m ; \Gamma^{\infty}\right)$, where $t$ is a positive real number, $p, q$ are two real numbers not smaller than 1 and $m$ is an integer ( $m$ is the order of the Haar wavelets used for constructing the space). Here $J$ stands for jumps, since the considered functions may jump at the multiple points of $\Gamma^{\infty}$. Note that if a self-similar set $\mathcal{S}$ is totally disconnected, then the JLip spaces on $\mathcal{S}$ coincide with Lipschitz or Besov spaces, more precisely that the spaces $J \operatorname{Lip}(t, p, q ; m ; \mathcal{S})$ coincide with the Lipschitz spaces $\operatorname{Lip}(t, p, q ; m ; \mathcal{S})$ also introduced in [12]. The latter are a generalization of the more classical spaces $\operatorname{Lip}(t, p, q ; \mathcal{S})$ introduced in [14] since $\operatorname{Lip}(t, p, q ;[t] ; \mathcal{S})=\operatorname{Lip}(t, p, q ; \mathcal{S})$. Note that $\operatorname{Lip}(t, p, q ; \mathcal{S})$ coincides with the Besov space $B_{t}^{p, q}(\mathcal{S})$, see[15]. Remember that if $p=q$ and $s \in(0,1)$

$$
B_{s}^{p, p}\left(\Gamma^{\infty}\right)=\left\{f \in L_{\mu}^{p}\left(\Gamma^{\infty}\right) ; \iint_{x, y \in \Gamma^{\infty},|x-y|<1} \frac{|f(x)-f(y)|^{p}}{|x-y|^{d+s p}} d \mu(x) d \mu(y)<\infty\right\} .
$$

- As a consequence, if $1 / 2 \leq a<a^{*}$, i.e. if $\Gamma^{\infty}$ is totally disconnected, then the trace space $\ell^{\infty}\left(W^{1, q}(\Omega)\right)$ is exactly $B_{1-\frac{2-d}{q}}^{q, q}\left(\Gamma^{\infty}\right)$. Note that $B_{1-\frac{2-d}{q}}^{q, q}\left(\Gamma^{\infty}\right)$ is precisely the image of $W^{1, q}\left(\mathbb{R}^{2}\right)$ by the classical trace operator, see [14].

- In the case when $a=a^{*}$, i.e. when the set $\Gamma^{\infty}$ self-intersects, relations between JLip and classical Besov spaces on $\Gamma^{\infty}$ were studied in [1]. As a consequence, it was also shown in 
[1] that there exits a critical exponent $\bar{q}_{\theta} \in(1,2)$, depending on the Hausdorff dimension of the self intersection of $\Gamma^{\infty}$ such that for all $q$ such that $1<q<\bar{q}_{\theta}$, the identity $\ell^{\infty}\left(W^{1, q}(\Omega)\right)=B_{1-\frac{2-d}{q}}^{q, q}\left(\Gamma^{\infty}\right)$ holds, and is false for $q>\bar{q}_{\theta}$.

The question of extensions is closely related to that of traces.

- Jones [11] (and Vodopjanov et al. [22] in the case $n=2$, see also [18, 14]) have studied the open bounded subsets $\Omega$ of $\mathbb{R}^{n}$ such that there exists a continuous extension operator from $W^{\ell, p}(\Omega)$ in $W^{\ell, p}\left(\mathbb{R}^{n}\right)$, for all nonnegative integers $\ell$ and real numbers $p, 1 \leq p \leq \infty$. Such domains are sometimes called Sobolev extension domains. Jones has proved that if $\Omega$ is an $\epsilon-\delta$ domain for some parameters $\epsilon, \delta>0$, see $[11,14]$ for the definition, then it is a Sobolev extension domain. Moreover, in dimension two, if $\Omega$ is finitely connected and a Sobolev extension domain, then $\Omega$ is an $\epsilon-\delta$ domain for some parameters $\epsilon, \delta>0$. In dimension two, the definition of such domains is equivalent to that of quasi-disks, see [18].

- It can be seen that if $1 / 2 \leq a<a^{*}$, the domain $\Omega$ is an $\epsilon-\delta$ domain, hence a Sobolev extension domain.

- If on the contrary $a=a^{*}$ then the domain $\Omega$ is not a Sobolev extension domain (it is easily seen that for $p>2$, the extension property from $W^{1, p}(\Omega)$ to $W^{1, p}\left(\mathbb{R}^{2}\right)$ is not satisfied; in this case, it was proved in [8] that $\Omega$ has the Sobolev extension property (from $W^{1, q}(\Omega)$ to $W^{1, q}\left(\mathbb{R}^{2}\right)$ ) for $1<q<\bar{q}_{\theta}$, where $\bar{q}_{\theta}$ is the critical exponent mentioned above; the proof of this result consisted in the construction of an extension operator which was compatible with that of $\ell^{\infty}$; it used the above-mentioned characterization of $\ell^{\infty}\left(W^{1, p}(\Omega)\right)$, and a strenghtened Poincaré inequality proved in [4]. The results obtained in [8] are recalled in $\S 3.5$ below.

A question that was not tackled in the previously mentioned works is the relation between the classically defined trace operator and the operator $\ell^{\infty}$ constructed using the self-similarity. Is it true that for a given function $v \in W^{1, p}(\Omega)$, the two definitions of the trace of $v$ on $\Gamma^{\infty}$ coincide $\mu$-almost everywhere on $\Gamma^{\infty}$ ? The goal of the present work is to give a positive answer to this question, using the extension operators constructed in [8]. As a consequence, we shall see in $\S 4.3$ that for $q>\bar{q}_{\theta}$, the domain $\Omega$ does not have the $W^{1, q}$ extension property.

\section{The Geometry}

\subsection{The similitudes $f_{1}$ and $f_{2}$ and the self-similar set $\Gamma^{\infty}$}

Consider four real numbers $a, \alpha, \beta, \theta$ such that $0<a<1 / \sqrt{2}, \alpha>0, \beta>0$ and $0<\theta<\pi / 2$. Let $f_{i}, i=1,2$ be the two similitudes in $\mathbb{R}^{2}$ given by

$$
\begin{aligned}
& f_{1}\left(\begin{array}{l}
x_{1} \\
x_{2}
\end{array}\right)=\left(\begin{array}{c}
-\alpha \\
\beta
\end{array}\right)+a\left(\begin{array}{c}
x_{1} \cos \theta-x_{2} \sin \theta \\
x_{1} \sin \theta+x_{2} \cos \theta
\end{array}\right), \\
& f_{2}\left(\begin{array}{l}
x_{1} \\
x_{2}
\end{array}\right)=\left(\begin{array}{c}
\alpha \\
\beta
\end{array}\right)+a\left(\begin{array}{c}
x_{1} \cos \theta+x_{2} \sin \theta \\
-x_{1} \sin \theta+x_{2} \cos \theta
\end{array}\right) .
\end{aligned}
$$


The two similitudes have the same dilation ratio $a$ and opposite angles $\pm \theta$. One can obtain $f_{2}$ by composing $f_{1}$ with the symmetry with respect to the axis $\left\{x_{1}=0\right\}$.

We denote by $\Gamma^{\infty}$ the self-similar set associated to the similitudes $f_{1}$ and $f_{2}$, i.e. the unique compact subset of $\mathbb{R}^{2}$ such that

$$
\Gamma^{\infty}=f_{1}\left(\Gamma^{\infty}\right) \cup f_{2}\left(\Gamma^{\infty}\right)
$$

For $n \geq 1$, we denote by

- $\mathcal{A}_{n}$ the set containing all the $2^{n}$ mappings from $\{1, \ldots, n\}$ to $\{1,2\}$

- $\mathcal{A}$ the set defined by $\mathcal{A}=\cup_{n \geq 1} \mathcal{A}_{n}$

- $\mathcal{A}_{\infty}=\{1,2\}^{\mathbb{N} \backslash\{0\}}$ the set of the sequences $\sigma=(\sigma(i))_{i=1, \ldots, \infty}$ with values $\sigma(i) \in\{1,2\}$.

Consider $1 \leq m<n \leq \infty$ and $\sigma \in \mathcal{A}_{n}$ : we say that $\sigma_{m} \in \mathcal{A}_{m}$ defined by $\sigma_{m}(i)=\sigma(i)$, $i=1, \ldots, m$ is a prefix of $\sigma$.

For a positive integer $n$ and $\sigma \in \mathcal{A}_{n}$, we define the similitude $f_{\sigma}$ by

$$
f_{\sigma}=f_{\sigma(1)} \circ \ldots \circ f_{\sigma(n)} .
$$

Similarly, if $\sigma \in \mathcal{A}_{\infty}$,

$$
f_{\sigma}=\lim _{n \rightarrow \infty} f_{\sigma(1)} \circ \ldots \circ f_{\sigma(n)}=\lim _{n \rightarrow \infty} f_{\sigma_{n}}
$$

Let the subset $\Gamma^{\infty, \sigma}$ of $\Gamma^{\infty}$ be defined by

$$
\Gamma^{\infty, \sigma}=f_{\sigma}\left(\Gamma^{\infty}\right)
$$

The definition of $\Gamma^{\infty}$ implies that for all $n>0, \Gamma^{\infty}=\bigcup_{\sigma \in \mathcal{A}_{n}} \Gamma^{\infty, \sigma}$. We also define the set $\Xi$ :

$$
\Xi=f_{1}\left(\Gamma^{\infty}\right) \cap f_{2}\left(\Gamma^{\infty}\right) \text {. }
$$

The following theorem was stated by Mandelbrot et al., [17] (a complete proof is given in [7]):

Theorem 1 For any $\theta, 0<\theta<\pi / 2$, there exists a unique positive number $a^{*}(\theta)<1 / \sqrt{2}$, (which does not depend of $(\alpha, \beta)$ see [4]) such that

$$
\begin{array}{rlll}
0<a<a^{*}(\theta) & \Rightarrow \Xi=\emptyset & \Rightarrow & \Gamma^{\infty} \text { is totally disconnected, } \\
a=a^{*}(\theta) & \Rightarrow \Xi \neq \emptyset & \Rightarrow & \Gamma^{\infty} \text { is connected, (from Th. 1.6.2 in [16]). }
\end{array}
$$

The critical parameter $a^{*}(\theta)$ is the unique positive root of the polynomial equation:

$$
\sum_{i=0}^{\mathfrak{m}-1} X^{i+2} \cos i \theta=\frac{1}{2},
$$

where

$$
\mathfrak{m} \text { is the smallest integer such that } \mathfrak{m} \theta \geq \pi / 2 \text {. }
$$

Remark 1 From (8), it can be seen that $\theta \mapsto a^{*}(\theta)$ is a continuous and increasing function from $(0, \pi / 2)$ onto $(1 / 2,1 / \sqrt{2})$ and that $\lim _{\theta \rightarrow 0} a^{*}(\theta)=1 / 2$.

Hereafter, for a given $\theta, 0<\theta<\pi / 2$, we will write for brevity $a^{*}$ instead of $a^{*}(\theta)$ and we will only consider $a$ such that $0<a \leq a^{*}$. 


\subsection{Ramified domains}

\subsubsection{The construction}

Call $P_{1}=(-1,0)$ and $P_{2}=(1,0)$ and $\Gamma^{0}$ the line segment $\Gamma^{0}=\left[P_{1}, P_{2}\right]$. We impose that $f_{2}\left(P_{1}\right)$, and $f_{2}\left(P_{2}\right)$ have positive coordinates, i.e. that

$$
a \cos \theta<\alpha \quad \text { and } \quad a \sin \theta<\beta .
$$

We also impose that the open domain $Y^{0}$ inside the closed polygonal line joining the points $P_{1}$, $P_{2}, f_{2}\left(P_{2}\right), f_{2}\left(P_{1}\right), f_{1}\left(P_{2}\right), f_{1}\left(P_{1}\right), P_{1}$ in this order is convex. With (10), this is true if and only if

$$
(\alpha-1) \sin \theta+\beta \cos \theta \geq 0 .
$$

Under assumptions (10) and (11), the domain $Y^{0}$ is either hexagonal or trapezoidal in degenerate cases, contained in the half-plane $x_{2}>0$ and symmetric w.r.t. the vertical axis $x_{1}=0$.

We introduce $K^{0}=\overline{Y^{0}}$. It is possible to glue together $K^{0}, f_{1}\left(K^{0}\right)$ and $f_{2}\left(K^{0}\right)$ and obtain a new polygonal domain, also symmetric with respect to the axis $\left\{x_{1}=0\right\}$. The assumptions (10) and (11) imply that $Y^{0} \cap f_{1}\left(Y^{0}\right)=\emptyset$ and $Y^{0} \cap f_{2}\left(Y^{0}\right)=\emptyset$. We also define the ramified open domain $\Omega$, see Figure 1:

$$
\Omega=\operatorname{Interior}\left(K^{0} \cup\left(\underset{\sigma \in \mathcal{A}}{\cup} f_{\sigma}\left(K^{0}\right)\right)\right) .
$$

Note that $\Omega$ is symmetric with respect to the axis $x_{1}=0$, and that for $a<1 / \sqrt{2}$, the measure of $\Omega$ is finite.

For a given $\theta$, with $a^{*}$ defined as above, we shall make the following assumption on $(\alpha, \beta)$ :

Assumption 1 For $0<\theta<\pi / 2$, the parameters $\alpha$ and $\beta$ satisfy (11) and (10) for $a=a^{*}$, and are such that

$$
\left\{\begin{array}{l}
\text { i) for all } a, 0<a \leq a^{*}, \text { the sets } Y^{0}, f_{\sigma}\left(Y^{0}\right), \sigma \in \mathcal{A}_{n}, n>0 \text {, are disjoint } \\
\text { ii) for all } a, 0<a<a^{*}, f_{1}(\bar{\Omega}) \cap f_{2}(\bar{\Omega})=\emptyset \\
\text { iii) for } a=a^{*}, f_{1}(\bar{\Omega}) \cap f_{2}(\bar{\Omega}) \neq \emptyset .
\end{array}\right.
$$

Remark 2 As proved in [4], Assumption 1 implies that if $a=a^{*}$, then $f_{1}(\Omega) \cap f_{2}(\Omega)=\emptyset$.

The following theorem asserts that $\forall \theta, 0<\theta<\pi / 2$, there exists $(\alpha, \beta)$ satisfying Assumption 1 .

Theorem 2 see [4]. If $\theta \in(0, \pi / 2)$, then for all $\alpha>a^{*} \cos \theta$, there exists $\bar{\beta}>0$ such that $\bar{\beta}>a^{*} \sin \theta$ and $(\alpha-1) \sin \theta+\bar{\beta} \cos \theta \geq 0$ and for all $\beta \geq \bar{\beta},(\alpha, \beta)$ satisfies Assumption 1 .

It has been proved in [3] that if $a<a^{*}$, then there exists $\epsilon>0$ and $\delta>0$ such that $\Omega$ is an $\epsilon-\delta$ domain as defined by Jones [11], see also [14] or in an equivalent manner a quasi-disk, see [18]. On the contrary, if $a=a^{*}$, then $\Omega$ is not an $\epsilon-\delta$ domain because it is possible to construct two sequences $\left(x_{n}^{(1)}\right)_{n}$ and $\left(x_{n}^{(2)}\right)_{n}, x_{n}^{(1)} \in f_{1}(\Omega)$ and $x_{n}^{(2)} \in f_{2}(\Omega)$ such that $\lim _{n \rightarrow \infty}\left|x_{n}^{(1)}-x_{n}^{(2)}\right|=0$; then, any arc contained in $\Omega$ and joining $x_{n}^{(1)}$ to $x_{n}^{(2)}$ has a length bounded from below by a positive constant. 


\subsubsection{The Moran condition}

The Moran condition, (or open set condition), see [20,16], is that there exists a nonempty bounded open subset $\omega$ of $\mathbb{R}^{2}$ such that $f_{1}(\omega) \cap f_{2}(\omega)=\emptyset$ and $f_{1}(\omega) \cup f_{2}(\omega) \subset \omega$. For a given $\theta \in(0, \pi / 2)$, let $(\alpha, \beta)$ satisfy Assumption 1 ; for $0<a \leq a^{*}$, the Moran condition is satisfied with $\omega=\Omega$ because

- $f_{1}(\Omega) \cap f_{2}(\Omega)=\emptyset$, which stems from point ii) in Assumption 1 if $a<a^{*}$, and from Remark 2 if $a=a^{*}$;

- by construction of $\Omega$, we also have $f_{1}(\Omega) \cup f_{2}(\Omega) \subset \Omega$.

The Moran condition implies that the Hausdorff dimension of $\Gamma^{\infty}$ is

$$
\operatorname{dim}_{H}\left(\Gamma^{\infty}\right)=d \equiv-\frac{\log 2}{\log a}
$$

see $[20,16]$. If $0<\theta<\pi / 2$, we have $0<a \leq a^{*}<1 / \sqrt{2}$ and thus $d<2$.

\subsection{3 $\Omega$ is a two-set}

The definition of a $d$-set is given in [14], chapter 8:

Definition 1 Let $E \subset \mathbb{R}^{2}$ be an arbitrary Borel set. Let $m_{d}$ be the d-dimensional Hausdorff measure. The set $E$ is a d-set if there exist three positive constants $r_{0}, c_{1}$ and $c_{2}$ such that for any closed ball $B(P, r), P \in E, 0<r \leq r_{0}$,

$$
c_{1} r^{d} \leq m_{d}(B(P, r) \cap E) \leq c_{2} r^{d} .
$$

Lemma 1 There exist two positive constants $\tilde{c}_{1}$ and $\tilde{c}_{2}$ such that for any $P \in \Gamma^{\infty}$ and for any $r, 0<r \leq \operatorname{diam}(\Omega)$,

$$
\tilde{c}_{1} r^{2} \leq m(B(P, r) \cap \Omega) \leq \tilde{c}_{2} r^{2}
$$

where $m=m_{2}$ is the standard Lebesgue measure un $\mathbb{R}^{2}$.

Proof. The right side of (15) is clearly true with $\tilde{c}_{2}=\pi$.

For the other inequality, take $P \in \Gamma^{\infty}$ and $r \in(0$, diam $\Omega]$. There exists $\sigma \in \mathcal{A}_{\infty}$ such that $P=$ $f_{\sigma}(P)$. Take $n \in \mathbb{N}$ such that $a^{n} \operatorname{diam} \Omega<r \leq a^{n-1} \operatorname{diam} \Omega$, which implies that diam $f_{\sigma_{n}}(\Omega)=$ $a^{n}$ diam $\Omega<r$. Since $P \in f_{\sigma_{n}}\left(\Gamma^{\infty}\right)$, one has $P \in \overline{f_{\sigma_{n}}(\Omega)}$, and we deduce that $f_{\sigma_{n}}(\Omega) \subset B(P, r)$. Therefore, since $f_{\sigma_{n}}(\Omega) \subset \Omega$,

$$
m(B(P, r) \cap \Omega) \geq m\left(f_{\sigma_{n}}(\Omega)\right)=a^{2 n} m(\Omega) \geq \frac{a^{2} m(\Omega)}{\operatorname{diam}^{2} \Omega} r^{2},
$$

and we obtain the desired result.

Proposition $1 \Omega$ is a two-set.

Proof. Call $r_{0}=\operatorname{diam}(\Omega)$. 
Step 1 We are going to prove first that there exist two positive constants $c_{3}$ and $c_{4}$ such that for all $P \in \Omega$, for all $n \in \mathbb{N}$,

$$
c_{3} a^{2 n} \leq m\left(B\left(P, a^{n} r_{0}\right) \cap \Omega\right) \leq c_{4} a^{2 n} .
$$

Obviously, one can take $c_{4}=\pi r_{0}^{2}$. Let us proceed by induction on $n$ :

$n=0$ : from the self-similarity, at least one of the following assertion is true: $P \in Y^{0}$ or $\min _{Q \in \Gamma^{\infty}}|P-Q| \leq a r_{0}$. Indeed, if $P \notin Y^{0}$, then one can suppose without restriction that $P \in f_{1}(\bar{\Omega})$. In this case, $\min _{Q \in \Gamma^{\infty}}|P-Q|=\min _{Q \in f_{1}\left(\Gamma^{\infty}\right)}|P-Q|$, because for all $Q^{\prime} \in f_{2}\left(\Gamma^{\infty}\right)$, the symmetric $Q$ of $Q^{\prime}$ with respect to the vertical axis belongs to $f_{1}\left(\Gamma^{\infty}\right)$ and is such that $|P-Q| \leq\left|P-Q^{\prime}\right|$. Therefore, $\min _{Q \in \Gamma^{\infty}}|P-Q| \leq a r_{0}$.

- Since $Y^{0}$ is a polygonal domain of $\mathbb{R}^{2}$ there exists a positive constant $c$ such that for all $r \leq r_{0}$, and $Q \in \overline{Y^{0}}, m\left(B(Q, r) \cap Y^{0}\right) \geq c r^{2}$. Therefore, if $P \in \overline{Y^{0}}$, then for all $r$, $0<r \leq r_{0}$, we have $m(B(P, r) \cap \Omega) \geq c r^{2}$.

- If $\min _{Q \in \Gamma^{\infty}}|P-Q| \leq a r_{0}$, call $Q_{\min }$ a point in $\Gamma^{\infty}$ achieving the minimum: then $B\left(Q_{\min },(1-\right.$ a) $\left.r_{0}\right) \subset B\left(P, r_{0}\right)$. Therefore, from Lemma $1, m\left(B\left(P, r_{0}\right) \cap \Omega\right) \geq m\left(B\left(Q_{\min },(1-a) r_{0}\right) \cap \Omega\right) \geq$ $\tilde{c}_{1}(1-a)^{2} r_{0}^{2}$.

We can take $c_{3}=\min \left(\tilde{c}_{1}(1-a)^{2}, c\right) r_{0}^{2}$.

$n \rightarrow n+1$ : The induction hypothesis is (16) with the value of $c_{3}$ defined above. Take $P \in \Omega$.

- If $P \in \overline{Y^{0}}$, we have that $m\left(B\left(P, a^{n+1} r_{0}\right) \cap \Omega\right) \geq c r_{0}^{2} a^{2(n+1)} \geq c_{3} a^{2(n+1)}$.

- If $P \notin \overline{Y^{0}}$, we can assume without restriction that $P \in f_{1}(\Omega)$. There exists $Q \in \Omega$ such that $P=f_{1}(Q)$ : therefore $f_{1}\left(B\left(Q, a^{n} r_{0}\right) \cap \Omega\right) \subset B\left(P, a^{n+1} r_{0}\right) \cap \Omega$, and from the induction hypothesis, $m\left(B\left(Q, a^{n} r_{0}\right) \cap \Omega\right) \geq c_{3} a^{2 n}$. Thus, $m\left(B\left(P, a^{n+1} r_{0}\right) \cap \Omega\right) \geq c_{3} a^{2(n+1)}$.

Step 2 For $0<r \leq r_{0}$, there exists a unique $n>0$ such that $r_{0} a^{n}<r \leq r_{0} a^{n-1}$. Thus, for all $p \in \Omega, m(B(p, r) \cap \Omega) \geq m\left(B\left(p, r_{0} a^{n}\right) \cap \Omega\right) \geq c_{3} a^{2 n} \geq \frac{c_{3} a^{2}}{r_{0}^{2}} r^{2}$. We have proved (14) with $c_{1}=\frac{c_{3} a^{2}}{r_{0}^{2}}$ and $c_{2}=\pi$.

\subsection{Hausdorff dimension of $\Xi$}

We aim at characterizing $\Xi$ defined in (6). We already know that $\Xi \neq \emptyset$ if and only if $a=a^{*}$. Let us denote by $\Lambda$ the vertical axis: $\Lambda=\left\{x: x_{1}=0\right\}$ and by $O$ the origin $O=(0,0)$. Since $f_{1}\left(\Gamma^{\infty}\right)=\Gamma^{\infty} \cap\left\{x_{1} \leq 0\right\}$ and $f_{2}\left(\Gamma^{\infty}\right)=\Gamma^{\infty} \cap\left\{x_{1} \geq 0\right\}$, we immediately see that $\Xi=\Gamma^{\infty} \cap \Lambda$. It can be observed (see [7] for the proof) that the sequences $\sigma \in \mathcal{A}_{\infty}$ such that $f_{\sigma}(O) \in \Lambda$ and that $\sigma(1)=1$ are characterized by the following property: for all $n \leq 1$, the truncated sequence $\sigma_{n}$ achieves the maximum of the abscissa of $f_{\eta}(O)$ over all $\eta \in \mathcal{A}_{n}$ such that $\eta(1)=1$.

The Hausdorff dimension of $\Xi$ depends on the value of $\mathfrak{m}$ defined in (9):

Proposition 2 see [17] and also [7]. If $\mathfrak{m} \theta>\pi / 2$ and $a=a^{*}$, then $\Xi$ contains a single point. If $\mathfrak{m} \theta=\pi / 2$ and $a=a^{*}$, then the Hausdorff dimension of $\Xi$ is $d / 2$, where $d$ is defined by (13). 


\subsection{Examples}

We make the choice $\theta=\pi / 4, \alpha=1-a / \sqrt{2}, \beta=1+a / \sqrt{2}$. Hence $\mathfrak{m}=2$. The critical parameter $a^{*}(\pi / 4)$ is the unique positive solution of $X^{3}+\sqrt{2} X^{2}-\sqrt{2} / 2=0$, i.e. $a \leq a^{*}(\pi / 4) \simeq 0.593465$. The construction described in $\S 2.2 .1$ with the critical value $a=a^{*}(\pi / 4)$ leads to the domain $\Omega$ shown in the left part of Figure 1. If $a>1 / 2$, the Hausdorff dimension of $\Gamma^{\infty}$ is larger than one. For instance, if $a=a^{*}(\pi / 4)$, then $\operatorname{dim}_{H}\left(\Gamma^{\infty}\right) \simeq 1.3284371$. In the right part of Figure 1, we show a similar construction with $\theta=\pi / 5$ (for which $\mathfrak{m}=3$ ) and $a=a^{*}(\pi / 5) \simeq 0.56658$. Note the difference between the two cases: in the former case $\mathfrak{m}(\theta) \cdot \theta=\pi / 2$ and the set $\Xi$ defined in (6) is not countable whereas in the latter case, $\mathfrak{m}(\theta) \cdot \theta>\pi / 2$ and the set $\Xi$ is a singleton.
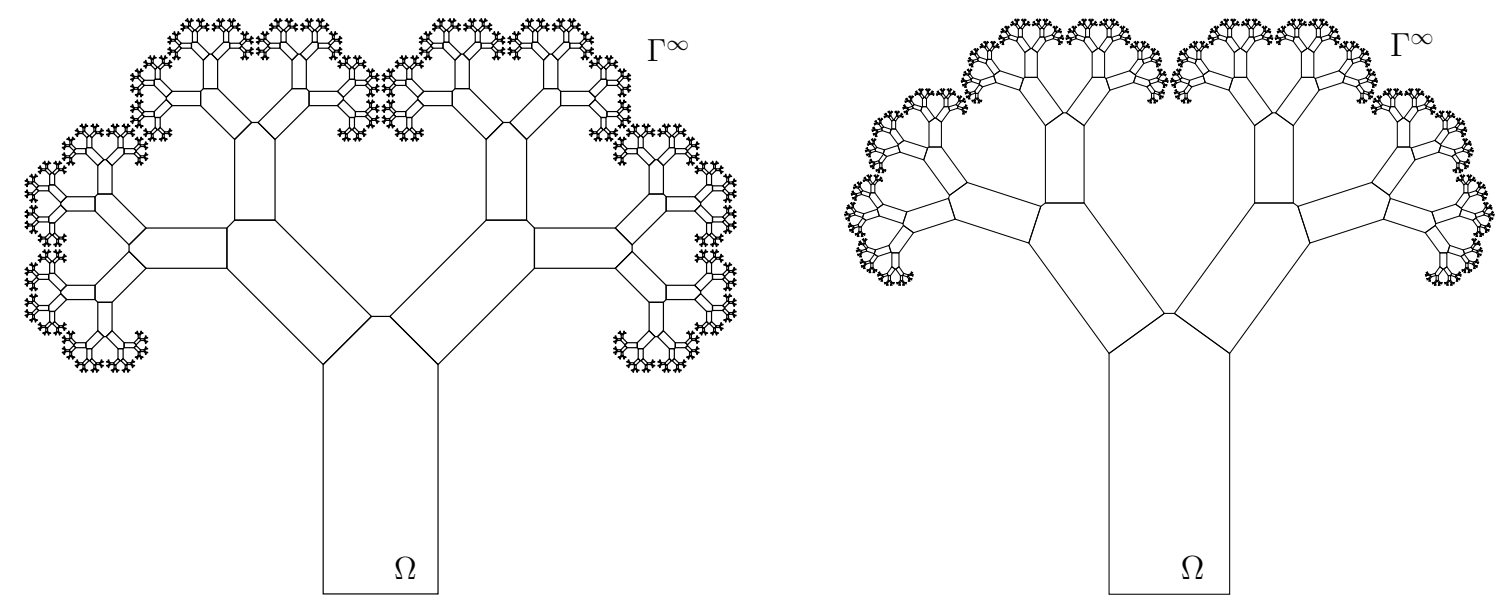

Figure 1: Left: the ramified domain $\Omega$ for $\theta=\pi / 4, a=a^{*}(\pi / 4), \alpha=1-a^{*} / \sqrt{2}, \beta=1+a^{*} / \sqrt{2}$. Right: a similar construction for $\theta=\pi / 5$ and $a=a^{*}(\pi / 5)$.

\subsection{The self-similar measure $\mu$}

To define traces on $\Gamma^{\infty}$, we recall the classical result on self-similar measures, see $[9,10]$ and $[16]$ page 26:

Theorem 3 There exists a unique Borel regular probability measure $\mu$ on $\Gamma^{\infty}$ such that for any Borel set $A \subset \Gamma^{\infty}$,

$$
\mu(A)=\frac{1}{2} \mu\left(f_{1}^{-1}(A)\right)+\frac{1}{2} \mu\left(f_{2}^{-1}(A)\right) .
$$

The measure $\mu$ is called the self-similar measure defined in the self-similar triplet $\left(\Gamma^{\infty}, f_{1}, f_{2}\right)$.

Proposition 3 The measure $\mu$ is a d-measure on $\Gamma^{\infty}$, with $d=-\log 2 / \log a$, according to the definition in [14], page 28: there exist two positive constants $c_{1}$ and $c_{2}$ such that

$$
c_{1} r^{d} \leq \mu\left(B(x, r) \cap \Gamma^{\infty}\right) \leq c_{2} r^{d}
$$


for any $r 0<r<1$ and $x \in \Gamma^{\infty}$, where $B(x, r)$ is the Euclidean ball in $\mathbb{R}^{2}$ centered at $x$ and with radius $r$.

Proof. The proof stems from the Moran condition. It is due to Moran [20] and has been extended by Kigami, see [16], §1.5, especially Proposition 1.5.8 and Theorem 1.5.7.

\subsection{Additional notations}

We define the sets $\Gamma^{\sigma}=f_{\sigma}\left(\Gamma^{0}\right)$ and $\Gamma^{N}=\cup_{\sigma \in \mathcal{A}_{N}} \Gamma^{\sigma}$. The one-dimensional Lebesgue measure of $\Gamma^{\sigma}$ for $\sigma \in \mathcal{A}_{N}$ and of $\Gamma^{N}$ are

$$
\left|\Gamma^{\sigma}\right|=a^{N}\left|\Gamma^{0}\right| \text { and }\left|\Gamma^{N}\right|=(2 a)^{N}\left|\Gamma^{0}\right| \text {. }
$$

\section{The space $W^{1, q}(\Omega)$}

Hereafter, we take $\theta$ in $(0, \pi / 2)$ and suppose that the parameters $(\alpha, \beta)$ satisfy Assumption 1 .

\subsection{Basic facts}

For a real number $q \geq 1$, let $W^{1, q}(\Omega)$ be the space of functions in $L^{q}(\Omega)$ with first order partial derivatives in $L^{q}(\Omega)$. The space $W^{1, q}(\Omega)$ is a Banach space with the norm

$\left(\|u\|_{L^{q}(\Omega)}^{q}+\left\|\frac{\partial u}{\partial x_{1}}\right\|_{L^{q}(\Omega)}^{q}+\left\|\frac{\partial u}{\partial x_{2}}\right\|_{L^{q}(\Omega)}^{q}\right)^{\frac{1}{q}}$, see for example [5], p 60. Elementary calculus shows that $\|u\|_{W^{1, q}(\Omega)} \equiv\left(\|u\|_{L^{q}(\Omega)}^{q}+\|\nabla u\|_{L^{q}(\Omega)}^{q}\right)^{\frac{1}{q}}$ is an equivalent norm, with $\|\nabla u\|_{L^{q}(\Omega)}^{q} \equiv \int_{\Omega}|\nabla u|^{q}$ and $|\nabla u|=\sqrt{\left|\frac{\partial u}{\partial x_{1}}\right|^{2}+\left|\frac{\partial u}{\partial x_{2}}\right|^{2}}$.

The spaces $W^{1, q}(\Omega)$ as well as elliptic boundary value problems in $\Omega$ have been studied in [2], with, in particular Poincaré inequalities and a Rellich compactness theorem. The same results in a similar but different geometry were proved by Berger [6] with other methods.

\subsection{The classical definition of traces}

We recall the classical definition of a trace operator on $\partial \omega$, see for instance [14] page 206, when $\omega$ is an open subset of $\mathbb{R}^{2}$.

Definition 2 Consider an open set $\omega \subset \mathbb{R}^{2}$. The function $u \in L_{l o c}^{1}(\omega)$ can be strictly defined at $x \in \bar{\omega}$ if the limit

$$
\bar{u}(x)=\lim _{r \rightarrow 0} \frac{1}{m_{2}(B(x, r) \cap \omega)} \int_{B(x, r) \cap \omega} u(z) d z
$$

exists.

The trace $\left.u\right|_{\partial \omega}$ is defined as the function given by $\left.u\right|_{\partial \omega}(x)=\bar{u}(x)$ at every point $x \in \partial \omega$ such that the limit $\bar{u}(x)$ exists.

In [14], Jonsson and Wallin proved the following result, ([14] page 206 Prop. 2), which we state in the particular case when the space dimension is two: 
Proposition 4 Let $\omega$ be an open 2-set such that the boundary $\partial \omega$ of $\omega$ is a 1-set. Let us assume that for a real number $q \in(1, \infty)$, there exists an extension operator $\mathcal{E}: W^{1, q}(\omega) \rightarrow W^{1, q}\left(\mathbb{R}^{2}\right)$. Let $u \in W^{1, q}(\omega)$, then $u$ can be strictly defined in $\partial \omega$ almost everywhere with respect to the one-dimensional Hausdorff measure $m_{1}$ and $\bar{u}=\mathcal{E} u m_{1}-$ a.e..

Although $\Omega$ defined above is a two-set, Proposition 4 does not apply to $\Omega$ because $\partial \Omega$ is not a 1 -set even in the cases when $\Gamma^{\infty}$ is a 1 -set.

\subsection{A trace operator defined by self-similarity}

In the remaining part of the paper, we will take $a$ such that $1 / 2 \leq a \leq a^{*}$, so the Hausdorff dimension $d$ of $\Gamma^{\infty}$ is not smaller than 1 .

We define $L_{\mu}^{q}, q \in[1,+\infty)$ as the class of $\mu$-measurable functions $v$ on $\Gamma^{\infty}$ such that $\int_{\Gamma^{\infty}}|v|^{q} d \mu<$ $\infty$, endowed with the norm $\|v\|_{L_{\mu}^{q}}=\left(\int_{\Gamma^{\infty}}|v|^{q} d \mu\right)^{1 / q}$. We also introduce $L_{\mu}^{\infty}$, the class of essentially bounded functions with respect to the measure $\mu$. A Hilbertian basis of $L_{\mu}^{2}$ can be constructed with e.g. Haar wavelets.

We recall the construction of the trace operator made in [2] by taking advantage of the selfsimililarity; this trace operator, called $\ell^{\infty}$ below, is well defined even if $a=a^{*}$.

We first construct a sequence $\left(\ell^{n}\right)_{n}$ of approximations of the trace operator: consider the sequence of linear operators $\ell^{n}: W^{1, q}(\Omega) \rightarrow L_{\mu}^{q}$,

$$
\ell^{n}(v)=\sum_{\sigma \in \mathcal{A}_{n}}\left(\frac{1}{\left|\Gamma^{\sigma}\right|} \int_{\Gamma^{\sigma}} v d x\right) \mathbf{1}_{f_{\sigma}\left(\Gamma^{\infty}\right)},
$$

where $\left|\Gamma^{\sigma}\right|$ is the one-dimensional Lebesgue measure of $\Gamma^{\sigma}$.

Proposition 5 see [2]. The sequence $\left(\ell^{n}\right)_{n}$ converges in $\mathcal{L}\left(W^{1, q}(\Omega), L_{\mu}^{q}\right)$ to an operator that we call $\ell^{\infty}$.

The operator $\ell^{\infty}$ is clearly a trace operator on $\Gamma^{\infty}$. The range of $\ell^{\infty}$ has been characterized in [4]:

For a given $\theta, 0<\theta<\pi / 2$, let $(\alpha, \beta)$ satisfy Assumption 1 and $\Omega$ be constructed as in $\S 2.2 .1$, with $1 / 2 \leq a \leq a^{*}$; then for all $q, 1<q<\infty$,

$$
\ell^{\infty}\left(W^{1, q}(\Omega)\right)=J \operatorname{Lip}\left(1-\frac{2-d}{q}, q, q ; 0 ; \Gamma^{\infty}\right),
$$

see $\S 1$ for comments about the JLip spaces.

\subsection{Extension results in the case when $a<a^{*}$}

We have seen in $\S 1$ that if $\omega$ is an $\epsilon-\delta$ domain, then it is a Sobolev extension domain. In dimension $n=2$, the definition of such domains is equivalent to that of quasi-disks, see [18], and the extension operator can be chosen such that $\mathcal{E}(u) \geq 0$ if $u \geq 0$.

The above mentioned results clearly hold with our domains $\Omega$ if $a<a^{*}$. 


\subsection{An extension result in the case when $a=a^{*}$}

When $a=a^{*}(\theta), \Omega$ is not an $\epsilon-\delta$ domain, and the extension results of Jones and Vodopjanov et al. cannot not be used. In [8], T. Deheuvels has proved the following theorem:

Theorem 4 see [8]

1. If $\mathfrak{m} \theta>\frac{\pi}{2}$ and $q \in(1,2)$, then there exists a continuous linear operator $\mathcal{E}$ from $W^{1, q}(\Omega)$ to $W^{1, q}\left(\mathbb{R}^{2}\right)$ such that

$$
\left.\mathcal{E}(u)\right|_{\Omega}=u, \quad \forall u \in W^{1, q}(\Omega)
$$

and such that $\mathcal{E}(u) \geq 0$ is $u \geq 0$.

2. If $\mathfrak{m} \theta=\frac{\pi}{2}$ and $q \in\left(1,2-\frac{d}{2}\right)$, then there exists a continuous linear operator $\mathcal{E}$ from $W^{1, q}(\Omega)$ to $W^{1, q}\left(\mathbb{R}^{2}\right)$ such that

$$
\left.\mathcal{E}(u)\right|_{\Omega}=u, \quad \forall u \in W^{1, q}(\Omega)
$$

and such that $\mathcal{E}(u) \geq 0$ is $u \geq 0$.

Let $\bar{q}_{\theta}$ be defined by $\bar{q}_{\theta}=2$ if $\mathfrak{m} \theta>\frac{\pi}{2}$ and $\bar{q}_{\theta}=2-\frac{d}{2}$ if $\mathfrak{m} \theta>\frac{\pi}{2}$; it is clear that $\bar{q}_{\theta}>1$ and a consequence of Theorem 4 is that $\Omega$ has the $W^{1, q}$ extension property (thus $\left.W^{1, q}\left(\mathbb{R}^{2}\right)\right|_{\Omega}=$ $\left.W^{1, q}(\Omega)\right)$ if $q \in\left(1, \bar{q}_{\theta}\right)$.

The construction of $\mathcal{E}$ in [8] takes very much advantage of the self-similarity. It relies on the following Theorem.

Theorem 5 see [8] In the case when $a=a^{*}$, if $q<\bar{q}_{\theta}$, then

$$
\ell^{\infty}\left(\left.W^{1, q}\left(\mathbb{R}^{2}\right)\right|_{\Omega}\right)=\ell^{\infty}\left(W^{1, q}(\Omega)\right)=J \operatorname{Lip}\left(1-\frac{2-d}{q}, q, q ; 0 ; \Gamma^{\infty}\right) .
$$

Remark 3 In [14], Jonsson and Wallin proved that $\left.W^{1, q}\left(\mathbb{R}^{2}\right)\right|_{\Gamma^{\infty}}=B_{1-\frac{2-d}{q}}^{q, q}\left(\Gamma^{\infty}\right)$ for all $q \in$ $(1, \infty)$, where the trace is meant in the classical sense (Theorem 1 p. 183). Theorem 5 can be seen as the counterpart of this result for the functions in $W^{1, q}(\Omega)$, (note that the self-similar definition of the trace $\ell^{\infty}$ is used and also the limitation on $\left.q\right)$.

Note that the critical exponent $\bar{q}_{\theta}$ already appeared in [1], where it was proved if $a=a^{*}$ and $1<q<\bar{q}_{\theta}$, then

$$
\ell^{\infty}\left(W^{1, q}(\Omega)\right)=J \operatorname{Lip}\left(1-\frac{2-d}{q}, q, q ; 0 ; \Gamma^{\infty}\right)=\operatorname{Lip}\left(1-\frac{2-d}{q}, q, q ; 0 ; \Gamma^{\infty}\right)=B_{1-\frac{2-d}{q}}^{q, q}\left(\Gamma^{\infty}\right),
$$

and that the previous space identities do not hold for $q>\bar{q}_{\theta}$.

\section{Comparison of the two definitions of trace}

We consider $a$ such that $1 / 2 \leq a \leq a^{*}$. Hereafter, the notation $\bar{u}(x)$ will be used for the strict definition at $x \in \bar{\Omega}$ of a function $u \in L^{1}(\Omega)$. 


\subsection{Some results on the classically defined trace}

From $\S 3.4$ and $\S 3.5$, we know that there exists a linear extension operator $\mathcal{E}$ which is continuous from $W^{1, q}(\Omega)$ to $W^{1, q}\left(\mathbb{R}^{2}\right)$, for all $q, q \in\left(1, \bar{q}_{\theta}\right)$ and which preserves the signs of the functions, i.e. if $u \geq 0$ in $\Omega$ then $\mathcal{E}(u) \geq 0$ in $\mathbb{R}^{2}$. Note that if $1 / 2 \leq a<a^{*}$, then the extension property holds for all $q>1$, but we will not need this in what follows.

As a consequence, the operator $\mathcal{E}$ is continuous from $W^{1, p}(\Omega)$ to $W^{1, q}\left(\mathbb{R}^{2}\right)$, for all $p \geq \bar{q}_{\theta}$ and $1<q<\bar{q}_{\theta}$ and from $W^{1, p}(\Omega)$ to $W^{1, q}\left(\mathbb{R}^{2}\right)$, for all $q \leq p<\bar{q}_{\theta}$. This extension operator is an important ingredient for proving the following result:

Theorem 6 Let $p>1$ be a real number. For all $u \in W^{1, p}(\Omega)$, $u$ can be strictly defined $m_{1}$ almost everywhere on $\partial \Omega$. Furthermore, $m_{1}$-almost everywhere on $\partial \Omega, \bar{u}$ defined by (18) coincides with $\widetilde{\mathcal{E}(u)}$, the strictly defined function in $\mathbb{R}^{2}$ which is given by

$$
\widetilde{\mathcal{E}(u)}(x)=\lim _{r \rightarrow 0} \frac{1}{m_{2}(B(x, r))} \int_{B(x, r)} \mathcal{E}(u)(z) d z, \text { when the limit exists. }
$$

Proof. The proof is similar to that of proposition 2 in [14] page 206. It is based on some results on potential spaces, which we recall in the specific present framework for completeness.

Assume that $u$ is positive. This is not a restriction, since it is always possible to decompose $u$ in $u=u^{+}-u^{-}$with $u^{+} \geq 0, u^{-} \geq 0$ and $u^{+}, u^{-} \in W^{1, p}(\Omega)$. We know that $\mathcal{E}(u)$ is positive and belongs to $\in W^{1, q}\left(\mathbb{R}^{2}\right)$ for some $q, 1<q \leq \min \left(p, \bar{q}_{\theta}\right)$.

Thanks to the identification of the potential spaces and the Sobolev spaces (see for instance E.M. Stein [21] Theorem 3, Ch. V page 135), there exist $g \in L^{q}\left(\mathbb{R}^{2}\right)$, such that $\mathcal{E}(u)=G_{1} \star g$ where $G_{1}$ is the Bessel kernel of order one. Take $\beta \in(0,2)$. N.G. Meyers proved in [19], Theorem 3.2 page 165 that for almost every $\xi_{0}$ (with respect to the $\beta$-Hausdorff measure), there exists a set $E_{\xi_{0}}$ such that

1. $\mathcal{E}(u)\left(\xi_{0}\right)=\lim _{\xi \rightarrow \xi_{0}, \xi \notin E_{\xi_{0}}} \mathcal{E}(u)(\xi)$,

2. $\lim _{r \rightarrow 0} \frac{C_{q}\left(B\left(\xi_{0}, r\right) \cap E_{\xi_{0}}\right)}{r^{\beta}}=0$,

where $C_{q}(A)$ is the capacity of a Borel set $A$, which is defined by

$$
C_{q}(A)=\inf \left\{\int_{\mathbb{R}^{2}} f^{q}: f \geq 0 \text { on } \mathbb{R}^{2} \quad G_{1} \star f(x) \geq 1 \quad \forall x \in A\right\} .
$$

Moreover, thanks to Proposition 1 page 151 in [14], see also [13], page 178, $\mathcal{E}(u)$ coincides with $\widetilde{\mathcal{E}(u)}$ a.e. with respect to the $\beta$-Hausdorff measure if $1-\frac{(2-\beta)}{q}>0$.

For a fixed value of $\beta>2-q$, let us consider $\xi_{0} \in \mathbb{R}^{2}$ with the previously mentioned properties. Let $q^{*}$ be the Sobolev exponent associated to $q$ i.e. $q^{*}=\frac{2 q}{2-q}$ (we can always assume that $q<2)$ and $q^{*^{\prime}}$ the conjugate exponent of $q^{*}$. Then for any $f \in L^{q}\left(\mathbb{R}^{2}\right)$ such that $f \geq 0$ and $G_{1} \star f(x) \geq 1 \quad \forall x \in B\left(\xi_{0}, r\right) \cap E_{\xi_{0}}$

$$
\begin{aligned}
m_{2}\left(B\left(\xi_{0}, r\right) \cap E_{\xi_{0}}\right) & \leq \int_{B\left(\xi_{0}, r\right) \cap E_{\xi_{0}}} G_{1} \star f \leq\left\|G_{1} \star f\right\|_{L^{q^{*}\left(\mathbb{R}^{2}\right)}}\left(m_{2}\left(B\left(\xi_{0}, r\right) \cap E_{\xi_{0}}\right)\right)^{\frac{1}{q^{*^{\prime}}}} \\
& \leq c\|f\|_{L^{q}\left(\mathbb{R}^{2}\right)}\left(m_{2}\left(B\left(\xi_{0}, r\right) \cap E_{\xi_{0}}\right)\right)^{\frac{1}{q^{*^{\prime}}}}
\end{aligned}
$$


and

$$
m_{2}\left(B\left(\xi_{0}, r\right) \cap E_{\xi_{0}}\right)^{q / q^{*}} \leq c^{q}\|f\|_{L^{q\left(\mathbb{R}^{2}\right)}}^{q} .
$$

Then, minimizing with respect to $f$ and using N.G. Meyers result,

$$
m_{2}\left(B\left(\xi_{0}, r\right) \cap E_{\xi_{0}}\right)^{\frac{2-q}{2}} \leq C_{q} c^{q}\left(B\left(\xi_{0}, r\right) \cap E_{\xi_{0}}\right)=o\left(r^{\beta}\right),
$$

which implies that

$$
m_{2}\left(B\left(\xi_{0}, r\right) \cap E_{\xi_{0}}\right)=o\left(r^{\frac{2 \beta}{2-q}}\right) .
$$

Thanks to the assumptions on $\xi_{0}$,

$$
\begin{aligned}
\widetilde{\mathcal{E}(u)}\left(\xi_{0}\right) & =\lim _{r \rightarrow 0} \frac{1}{m_{2}\left(B\left(\xi_{0}, r\right)\right)} \int_{B\left(\xi_{0}, r\right)} \mathcal{E}(u) d m_{2} \\
& =\lim _{r \rightarrow 0} \frac{1}{m_{2}\left(B\left(\xi_{0}, r\right)\right)}\left(\int_{B\left(\xi_{0}, r\right) \cap E_{\xi_{0}}} \mathcal{E}(u) d m_{2}+\int_{B\left(\xi_{0}, r\right) \cap E_{\xi_{0}}^{c}} \mathcal{E}(u) d m_{2}\right) .
\end{aligned}
$$

Thanks to the continuity property and (21), if $\beta>2-q$ then

$$
\lim _{r \rightarrow 0} \frac{1}{m_{2}\left(B\left(\xi_{0}, r\right)\right)} \int_{B\left(\xi_{0}, r\right) \cap E_{\xi_{0}}^{c}} \mathcal{E}(u) d m_{2}=\mathcal{E}(u)\left(\xi_{0}\right),
$$

and

$$
\lim _{r \rightarrow 0} \frac{1}{m_{2}\left(B\left(\xi_{0}, r\right)\right)} \int_{B\left(\xi_{0}, r\right) \cap E_{\xi_{0}}} \mathcal{E}(u) d m_{2}=0 .
$$

Hence

$$
\widetilde{\mathcal{E}}(u)\left(\xi_{0}\right)=\lim _{r \rightarrow 0} \frac{1}{m_{2}\left(B\left(\xi_{0}, r\right)\right)} \int_{B\left(\xi_{0}, r\right) \cap E_{\xi_{0}}^{c}} \mathcal{E}(u) d m_{2} .
$$

We now have all the tools necessary to begin the proof:

$$
\begin{aligned}
& \frac{1}{m_{2}\left(B\left(\xi_{0}, r\right) \cap \Omega\right)} \int_{B\left(\xi_{0}, r\right) \cap \Omega} u d m_{2}=\frac{1}{m_{2}\left(B\left(\xi_{0}, r\right) \cap \Omega\right)} \int_{B\left(\xi_{0}, r\right) \cap \Omega} \mathcal{E}(u) d m_{2} \\
= & \frac{1}{m_{2}\left(B\left(\xi_{0}, r\right) \cap \Omega\right)}\left(\int_{B\left(\xi_{0}, r\right) \cap \Omega \cap E_{\xi_{0}}} \mathcal{E}(u) d m_{2}+\int_{B\left(\xi_{0}, r\right) \cap \Omega \cap E_{\xi_{0}}^{c}} \mathcal{E}(u) d m_{2}\right) .
\end{aligned}
$$

For the first integral, since $\Omega$ is a 2 -set:

$$
\begin{aligned}
\frac{1}{m_{2}\left(B\left(\xi_{0}, r\right) \cap \Omega\right)} \int_{B\left(\xi_{0}, r\right) \cap \Omega \cap E_{\xi_{0}}} \mathcal{E}(u) d m_{2} & \leq \frac{1}{m_{2}\left(B\left(\xi_{0}, r\right) \cap \Omega\right)} \int_{B\left(\xi_{0}, r\right) \cap E_{\xi_{0}}} \mathcal{E}(u) d m_{2} \\
& \leq C \frac{1}{m_{2}\left(B\left(\xi_{0}, r\right)\right)} \int_{B\left(\xi_{0}, r\right) \cap E_{\xi_{0}}} \mathcal{E}(u) d m_{2}
\end{aligned}
$$

and as $r \rightarrow 0$, this tends to zero if $\beta>2-q$ thanks to (22).

For the second integral, if $\beta>2-q$, using the continuity property of $\mathcal{E}(u)$ in $\Omega \cap E_{\xi_{0}}^{c}$ and again the fact that $\Omega$ is a 2 -set,

$$
\lim _{r \rightarrow 0} \frac{1}{m_{2}\left(B\left(\xi_{0}, r\right) \cap \Omega\right)} \int_{B\left(\xi_{0}, r\right) \cap \Omega \cap E_{\xi_{0}}^{c}} \mathcal{E}(u) d m_{2}=\mathcal{E}(u)\left(\xi_{0}\right) .
$$


Hence, for all $\xi_{0}$ chosen as above, the limit $\bar{u}\left(\xi_{0}\right)=\lim _{r \rightarrow 0} \frac{1}{m_{2}\left(B\left(\xi_{0}, r\right) \cap \Omega\right)} \int_{B\left(\xi_{0}, r\right) \cap \Omega} u(z) d z$ exists and

$$
\bar{u}\left(\xi_{0}\right)=\mathcal{E}(u)\left(\xi_{0}\right)=\widetilde{\mathcal{E}(u)}\left(\xi_{0}\right) .
$$

We can choose $\beta=1$ since with $q>1$, the condition $\beta>2-q$ is fulfilled. This concludes the proof.

\subsection{Self-similar strictly defined trace on $\Gamma^{\infty}$}

Recall that $\Xi=f_{1}\left(\Gamma^{\infty}\right) \cap f_{2}\left(\Gamma^{\infty}\right)$, and define the set of the multiple points of $\Gamma^{\infty}, \Xi^{\infty}=$ $\Xi \cup \bigcup_{n=1}^{\infty} \bigcup_{\sigma \in \mathcal{A}_{n}} f_{\sigma}(\Xi)$. Note that $\mu\left(\Xi^{\infty}\right)=0$ and $m_{1}\left(\left(\Xi^{\infty}\right)=0\right.$. For $x \in \Gamma^{\infty} \backslash \Xi^{\infty}$, there is one and only one $\tau_{x} \in \mathcal{A}_{\infty}$ such that for all $n>0, x \in f_{\tau_{x, n}}\left(\Gamma^{\infty}\right)$, where $\tau_{x, n} \in \mathcal{A}_{n}$ and $\tau_{x, n}(i)=\tau_{x}(i)$ for $i=1, \ldots, n$.

Definition 3 The function $u \in L_{\text {loc }}^{1}(\Omega)$ can be self-similarly strictly defined at $x \in \Gamma^{\infty} \backslash \Xi^{\infty}$ if the limit

$$
\lim _{n \rightarrow \infty} \frac{1}{m_{2}\left(\Omega^{\tau_{x, n}}\right)} \int_{\Omega^{\tau x, n}} u(z) d z
$$

exists.

The self-similar strictly defined trace of $u$ on $\Gamma^{\infty}$ is then defined as

$$
\check{\ell}^{\infty}(u)(x)=\lim _{n \rightarrow \infty} \frac{1}{m_{2}\left(\Omega^{\tau_{x, n}}\right)} \int_{\Omega^{\tau_{x, n}}} u(z) d z
$$

at every point $x \in \Gamma^{\infty} \backslash \Xi^{\infty}$ such that the limit exists.

Theorem 7 Let $p>1$ be a real number. For all $u \in W^{1, p}(\Omega)$, the limit in the right hand side of (24) can be self-similarly strictly defined $m_{1}$-almost everywhere on $\Gamma^{\infty}$. Furthermore, $m_{1}$-almost everywhere on $\Gamma^{\infty}, \check{\ell}^{\infty}(u)$ coincides with $\widetilde{\mathcal{E}(u)}$.

Proof. The proof is very similar to that of Theorem 6 and we take the same notation. We know that for $\beta=1$, we have $1-\frac{2-\beta}{q}=1-\frac{1}{q}>0$ where $q \in(1,2)$ is defined in the proof of Theorem 6. Hence, for almost every $\xi_{0} \in \Gamma^{\infty} \backslash \Xi^{\infty}$ w.r.t. $m_{1}$, there exists a $E_{\xi_{0}}$ such that

1. $\mathcal{E}(u)\left(\xi_{0}\right)=\lim _{\xi \rightarrow \xi_{0}, \xi \notin E_{\xi_{0}}} \mathcal{E}(u)(\xi)$,

2. $\lim _{r \rightarrow 0} \frac{C_{q}\left(B\left(\xi_{0}, r\right) \cap E_{\xi_{0}}\right)}{r}=0$,

and that $\mathcal{E}(u)$ coincides with $\widetilde{\mathcal{E}(u)}$ at $\xi_{0}$. Fix such a $\xi_{0}$. We write $\tau=\tau_{\xi_{0}}$ and $\tau_{n}=\tau_{\xi_{0}, n}$ for brevity.

We know that $\Omega^{\tau_{n}} \subset B\left(\xi_{0}, a^{n} \operatorname{diam}(\Omega)\right)$ and that $m_{2}\left(\Omega^{\tau_{n}}\right)=a^{2 n} m_{2}(\Omega)$.

$\frac{1}{m_{2}\left(\Omega^{\tau_{n}}\right)} \int_{\Omega^{\tau_{n}}} u d m_{2}=\frac{1}{m_{2}\left(\Omega^{\tau_{n}}\right)} \int_{\Omega^{\tau_{n}}} \mathcal{E}(u) d m_{2}=\frac{1}{m_{2}\left(\Omega^{\tau_{n}}\right)}\left(\int_{\Omega^{\tau_{n}} \cap E_{\xi_{0}}} \mathcal{E}(u) d m_{2}+\int_{\Omega^{\tau_{n}} \cap E_{\xi_{0}}^{c}} \mathcal{E}(u) d m_{2}\right)$. 
Let us call $r_{n}=a^{n} \operatorname{diam}(\Omega)$. For the first integral,

$$
\begin{aligned}
\frac{1}{m_{2}\left(\Omega^{\tau_{n}}\right)} \int_{\Omega^{\tau_{n}} \cap E_{\xi_{0}}} \mathcal{E}(u) d m_{2} & \leq \frac{m_{2}\left(B\left(\xi_{0}, r_{n}\right) \cap \Omega\right)}{m_{2}\left(\Omega^{\tau_{n}}\right)} \frac{1}{m_{2}\left(B\left(\xi_{0}, r_{n}\right) \cap \Omega\right)} \int_{B\left(\xi_{0}, r_{n}\right) \cap \Omega \cap E_{\xi_{0}}} \mathcal{E}(u) d m_{2} \\
& \leq \frac{\pi \operatorname{diam}^{2}(\Omega)}{m_{2}(\Omega)} \frac{1}{m_{2}\left(B\left(\xi_{0}, r_{n}\right) \cap \Omega\right)} \int_{B\left(\xi_{0}, r_{n}\right) \cap \Omega \cap E_{\xi_{0}}} \mathcal{E}(u) d m_{2},
\end{aligned}
$$

which tends to 0 as $n$ tends to infinity from the proof of Theorem 6 . The second term converges to $\mathcal{E}(u)\left(\xi_{0}\right)$ from $(21)$ and the continuity property of $\mathcal{E}(u)$ in $\Omega \cap E_{\xi_{0}}^{c}$.

\subsection{Comparison of the different traces}

Proposition 6 For any $u \in W^{1, p}(\Omega), 1<p<\infty$, there exists a set $\mathcal{N} \subset \Gamma^{\infty}$ containing $\Xi^{\infty}$ with $\mu(\mathcal{N})=0$ such that for all $x \in \Gamma^{\infty} \backslash \mathcal{N}$,

- $\widetilde{\mathcal{E}(u)}(x), \bar{u}(x)$ and $\left(\ell^{\infty}(u)\right)(x)$ are well defined,

- $\left(\ell^{\infty}(u)\right)(x)=\bar{u}(x)=\widetilde{\mathcal{E}(u)}(x)$.

Proof. From Theorem 6, we know that there exists a subset $\mathcal{N}_{1}$ of $\Gamma^{\infty}$ with $\mu\left(\mathcal{N}_{1}\right)=0$ such that $\widetilde{\mathcal{E}(u)}(x)$ and $\bar{u}(x)$ are well defined and coincide with each other for $x \in \Gamma^{\infty} \backslash \mathcal{N}_{1}$. From Theorem 7 , we know that there exists a subset $\mathcal{N}_{2}$ of $\Gamma^{\infty}$ containing $\Xi^{\infty}$ with $\mu\left(\mathcal{N}_{2}\right)=0$ such that $\widetilde{\mathcal{E}(u)}(x)$ and $\check{\ell}^{\infty}(u)(x)$ are well defined and coincide with each other for $x \in \Gamma^{\infty} \backslash \mathcal{N}_{2}$. Therefore, for $x \in \Gamma^{\infty} \backslash\left(\mathcal{N}_{1} \cup \mathcal{N}_{2}\right), \check{\ell}^{\infty}(u)(x), \widetilde{\mathcal{E}(u)}(x)$ and $\bar{u}(x)$ are well defined and coincide with each other. On the other hand, it is elementary to check that if $u \in W^{1, p}(\Omega)$ with $p \geq 1$, then

$$
\lim _{n \rightarrow \infty}\left\|\left(\ell^{n}(u)\right)(x)-\frac{1}{m_{2}\left(\Omega^{\tau_{n}(x)}\right)} \int_{\Omega^{\tau_{n}(x)}} u(z) d z\right\|_{L_{\mu}^{p}\left(\Gamma^{\infty}\right)}=0,
$$

because, for all $x \in \Gamma^{\infty} \backslash \Xi^{\infty}$,

$$
\left|\left(\ell^{n}(u)\right)(x)-\frac{1}{m_{2}\left(\Omega^{\tau_{n}(x)}\right)} \int_{\Omega^{\tau_{n}(x)}} u(z) d z\right| \leq c a^{n(1-2 / p)}\|\nabla u\|_{L^{p}\left(\Omega^{\tau_{n}(x)}\right)}
$$

and $\sum_{\tau \in \mathcal{A}_{n}} 2^{-n} a^{n(p-2)}\|\nabla u\|_{L^{p}\left(\Omega^{\tau}\right)}^{p}=\sum_{\tau} a^{n(p-2+d)}\|\nabla u\|_{L^{p}\left(\Omega^{\tau}\right)}^{p}$ tends to zero as $n$ tends to $+\infty$. This implies that $\ell^{\infty}(u)$ coincides with he $\check{\ell}^{\infty}(u) \mu$-almost everywhere on $\Gamma^{\infty}$.

As a consequence of the first point, $\ell^{\infty}(u)$ coincides with $\bar{u}$ and $\widetilde{\mathcal{E}(u)} \mu$-almost everywhere on $\Gamma^{\infty}$.

As a consequence of Proposition 6, we see that if $p>\bar{q}_{\theta}$, there does not exist any continuous extension operators from $W^{1, p}(\Omega)$ to $W^{1, p}\left(\mathbb{R}^{2}\right)$. Indeed, if such an extension operator $\mathcal{E}$ existed, then for any $u \in W^{1, p}\left(\Omega\right.$, the trace (in the classical sense) of $\mathcal{E}(u)$ on $\Gamma^{\infty}$, namely $\widetilde{\mathcal{E}(u)}$ would belong to $B_{1-\frac{2-d}{p}}^{p, p}\left(\Gamma^{\infty}\right)$ (see [14]), where $B_{s}^{p, p}\left(\Gamma^{\infty}\right)$ is given by $(1)$.

But since $\ell^{\infty}(u)$ and $\widetilde{\mathcal{E}(u)}$ coincide $\mu$-everywhere, this would imply that $\ell^{\infty}(u) \in B_{1-\frac{2-d}{p}}^{p, p}\left(\Gamma^{\infty}\right)$ for all $u \in W^{1, p}\left(\Omega\right.$. This is in contradiction with the characterization of $\ell^{\infty}\left(W^{1, p}(\Omega)\right)$ as JLip $(1-$ $\left.\frac{2-d}{p}, p, p ; 0 ; \Gamma^{\infty}\right)$ given in [4] and the fact that $J \operatorname{Lip}\left(1-\frac{2-d}{p}, p, p ; 0 ; \Gamma^{\infty}\right) \not \subset B_{1-\frac{2-d}{p}}^{p, p}\left(\Gamma^{\infty}\right)$ for $p>\bar{q}_{\theta}$, see $[1]$. 


\section{References}

[1] Y. Achdou, T. Deheuvels, and N. Tchou. JLip versus sobolev spaces on a class of self-similar fractal foliages. J. Math. Pures Appl. (9).

[2] Y. Achdou and N. Tchou. Neumann conditions on fractal boundaries. Asymptotic Analysis, 53(1-2):61-82, 2007.

[3] Y. Achdou and N. Tchou. Trace results on domains with self-similar fractal boundaries. J. Math. Pures Appl. (9), 89(6):596-623, 2008.

[4] Y. Achdou and N. Tchou. Trace theorems for a class of ramified domains with self-similar fractal boundaries. SIAM J. Math. Anal., 42(4):1449-1482, 2010.

[5] R.A. Adams and J.J.F. Fournier. Sobolev spaces, volume 140 of Pure and Applied Mathematics (Amsterdam). Elsevier/Academic Press, Amsterdam, second edition, 2003.

[6] G. Berger. Eigenvalue distribution of elliptic operators of second order with Neumann boundary conditions in a snowflake domain. Math. Nachr., 220:11-32, 2000.

[7] T. Deheuvels. PhD dissertation, in progress.

[8] T. Deheuvels. $W^{1, p}$-extension property for tree shaped domains with self-contacting fractal boundaries.

[9] K. Falconer. Techniques in fractal geometry. John Wiley \& Sons Ltd., Chichester, 1997.

[10] J.E. Hutchinson. Fractals and self-similarity. Indiana Univ. Math. J., 30(5):713-747, 1981.

[11] P.W. Jones. Quasiconformal mappings and extendability of functions in Sobolev spaces. Acta Math., 147(1-2):71-88, 1981.

[12] A. Jonsson. Haar wavelets of higher order on fractals and regularity of functions. J. Math. Anal. Appl., 290(1):86-104, 2004.

[13] A. Jonsson and H. Wallin. A Whitney extension theorem in $L_{p}$ and Besov spaces. Ann. Inst. Fourier (Grenoble), 28(1):vi, 139-192, 1978.

[14] A. Jonsson and H. Wallin. Function spaces on subsets of $\mathbf{R}^{n}$. Math. Rep., 2(1):xiv+221, 1984.

[15] A. Jonsson and H. Wallin. The dual of Besov spaces on fractals. Studia Math., 112(3):285300, 1995.

[16] J. Kigami. Analysis on fractals, volume 143 of Cambridge Tracts in Mathematics. Cambridge University Press, Cambridge, 2001.

[17] B.B. Mandelbrot and M. Frame. The canopy and shortest path in a self-contacting fractal tree. Math. Intelligencer, 21(2):18-27, 1999. 
[18] V.G. Maz'ja. Sobolev spaces. Springer Series in Soviet Mathematics. Springer-Verlag, Berlin, 1985. Translated from the Russian by T. O. Shaposhnikova.

[19] Norman G. Meyers. Continuity properties of potentials. Duke Math. J., 42:157-166, 1975.

[20] P. A. P. Moran. Additive functions of intervals and Hausdorff measure. Proc. Cambridge Philos. Soc., 42:15-23, 1946.

[21] Elias M. Stein. Singular integrals and differentiability properties of functions. Princeton Mathematical Series, No. 30. Princeton University Press, Princeton, N.J., 1970.

[22] S. K. Vodop'janov, V. M. Gol'dšteŭn, and T. G. Latfullin. A criterion for the extension of functions of the class $L_{2}^{1}$ from unbounded plane domains. Sibirsk. Mat. Zh., 20(2):416-419, 464, 1979. 